\title{
Comparison of Three High Resolution Real-Time Spectrometers for Microwave Ozone Profiling Instruments
}

\author{
Eric Sauvageat ${ }^{\circledR}$, Roland Albers ${ }^{\circledR}$, Mikko Kotiranta ${ }^{\circledR}$, Klemens Hocke ${ }^{\circledR}$, R. Michael Gomez, \\ Gerald E. Nedoluha ${ }^{\circledR}$, and Axel Murk ${ }^{(1)}$
}

\begin{abstract}
In this contribution, we present a comparison of three digital real-time spectrometers used in passive remote sensing of ozone and other trace gases in the middle atmosphere. During a period of six months, we connected the spectrometers to the same radiometric front-end to perform parallel observations of the ozone emission line at $110.836 \mathrm{GHz}$. This allowed us to better characterize a bias previously observed on the integrated spectra of the Acqiris AC240, a widely used digital spectrometer which has been used for more than a decade in many operational microwave radiometers. We investigated the bias under different atmospheric conditions and found that it is caused by multiple sources. Nonlinearities in the calibration are responsible for part of the bias, but a larger contribution stems from a second effect in the AC240. Although this error source is still partly unexplained, we found that a simple correction scheme simulating a spectral leakage can be applied to the integrated spectra of the AC240 and worked well on our range of observations. We also show that by applying our bias correction to the spectra, we can correct the bias in the ozone retrievals. There is still a need for further measurements to validate this approximate correction, but it could help to correct the numerous time series of ozone and other atmospheric constituents recorded by the AC240.
\end{abstract}

Index Terms-Atmospheric measurements, digital real-time spectrometers, microwave radiometry, microwave spectroscopy, ozone, remote sensing, water vapor.

\section{INTRODUCTION}

$\mathbf{M}$ ICROWAVE ground-based radiometers provide continuous, all-weather observations of many constituents in the atmosphere. Compared to satellites, they have a high temporal resolution and are therefore important for estimating long-term trends in atmospheric gas concentrations and for cross validating satellite observations. In particular, they have been used

Manuscript received May 30, 2021; revised August 25, 2021; accepted September 15, 2021. Date of publication September 22, 2021; date of current version October 15, 2021. The work of R. Michael Gomez and Gerald E. Nedoluha was supported in paty by NASA under the Upper Atmosphere Research Program and in part by the Office of Naval Research. (Corresponding author: Eric Sauvageat.)

Eric Sauvageat, Klemens Hocke, and Axel Murk are with the Institute of Applied Physics and the Oeschger Centre for Climate Change Research, University of Bern, 3012 Bern, Switzerland (e-mail: eric.sauvageat@iap.unibe.ch; klemens.hocke@iap.unibe.ch; axel.murk@iap.unibe.ch).

Roland Albers and Mikko Kotiranta are with the Institute of Applied Physics, University of Bern, 3012 Bern, Switzerland (e-mail: roland.albers@iap.unibe.ch; mikko.kotiranta@iap.unibe.ch).

R. Michael Gomez and Gerald E. Nedoluha are with the Remote Sensing Division, U.S. Naval Research Laboratory, Washington, DC 20375 USA (e-mail: mike.gomez@nrl.navy.mil; gerald.nedoluha@nrl.navy.mil).

Digital Object Identifier 10.1109/JSTARS.2021.3114446 successfully for monitoring ozone, water vapor, temperature, carbon monoxide, and winds in the middle atmosphere [1]-[4].

These instruments are particularly well suited for the observation of ozone in the stratosphere (the so-called "ozone layer") and in the lower mesosphere, i.e., from approximately 20 to $70 \mathrm{~km}$ altitude. Thanks to multiple ozone transition lines in the microwave frequency range and to the pressure broadening mechanism, it is possible to retrieve the vertical ozone mixing ratio profile in the stratosphere and the lower mesosphere through the so-called inversion problem [5]. Therefore, microwave radiometers have been used for ozone profiling in a variety of locations, especially since the Montreal Protocol (1987). Following the ban of the ozone depleting substances, the assessment of ozone recovery in the middle atmosphere has become a key scientific topic [6]. Due to their long lasting time series and their nearly continuous sampling capabilities, microwave ozone radiometers are important instruments to assess the ozone trends of the last two decades.

Today, most microwave radiometers for middle atmospheric sounding are using high-resolution real-time spectrometers to extract the spectral information from the atmospheric radio frequency (RF) signal. In the last decade, real-time digital spectrometers have progressively replaced the older acoustooptical (AOS), chirp-transform or filter bank (FB) spectrometers in radio astronomy and atmospheric remote sensing. Initially designed for radio astronomy, the Acqiris AC240 was the first commercially available spectrometer used in atmospheric profiling radiometers [7], [8]. Since then, it has been used in many instruments worldwide, in particular in ozone, temperature, and water vapor profiling radiometers. Despite the numerous time series collected with the AC240, its spectroscopic performance remains largely unknown and has not yet been quantified.

Different publications have shown a persistent negative bias in the time series derived from the AC240 compared to other datasets, but its origin has yet to be determined [9]-[12]. It concerns both ozone and water vapour instruments, which for most of them, are operated in the frame of the Network for the Detection of Atmospheric Composition Change (NDACC) [13]. Despite generally high uncertainties on passive radiometric observations, this persistent bias has raised some questions about the AC240 and some users have expressed the need for a more thorough investigation of the influence of this back-end on the measurements. 
Parallel measurements of atmospheric emission lines with different back-ends enable us to characterize their spectroscopic performance and quantify their influence on the atmospheric profiles. The only studies comparing the AC240 with other spectrometers were done at the time when the AC240 replaced older FB and AOS back-ends in some ozone radiometers, with the goal to homogenize the time series (using the AC240 as the reference) [14], [15]. These studies both show an altitude dependent bias (with a globally negative trend) from the time series derived from the AC240 compared to the older spectrometers. However, it has to be noted that the change from an FB or AOS to a high resolution FFT spectrometer also has other impacts on the time series (e.g., from the change in bandwidth or spectral resolution) that can influence the results of such comparisons significantly.

This study aims at presenting a first intercomparison between digital real-time spectrometers used in passive microwave remote sensing, including the AC240 and two state-of-the-art spectrometers. We compared the influence of these three digital back-ends on radiometric observations of the ozone emission line at $110.836 \mathrm{GHz}$. We organized a measurement campaign between January and June 2019 at the University of Bern and conducted a first set of analysis described in [16] and [17]. First results from this campaign suggested that the calibrated spectra from the AC240 was systematically biased, measuring a smaller ozone line amplitude and a different slope compared to the more recent spectrometers. To better characterize these discrepancies, we expanded on the previous analysis by investigating the sensitivity of the bias to the weather conditions and by quantifying its impact on the retrieved ozone profiles. We evaluated the effect of a simple correction scheme on the integrated spectra and the middle-atmospheric ozone profiles. In addition, we conducted new laboratory tests aiming to confirm the observed bias and help understand its origin.

The rest of this article is organized as follows. Section II describes the instrumental setup that was used during the measurement campaign and for the laboratory tests. Section III describes the measurement campaign and the associated data processing, from the calibration to the ozone retrievals. It presents and discusses the results of the campaign and their limitations. A discussion of the bias origin is made in Section IV, together with the latest laboratory investigations that we conducted to better understand it. Finally, a brief conclusion and outlook are presented in Section V.

\section{INSTRUMENTAL SETUP}

The microwave ozone profiling instruments (MOPI) are a series of ground-based radiometers operated by the United States Naval Research Laboratory (NRL) in the frame of NDACC. Since 1995, the MOPI instruments are monitoring ozone in the middle-atmosphere above Mauna Loa, Hawaii, and Lauder New Zealand [2].

As a front-end for our measurement campaign, we have used MOPI 5, which is a new, room temperature receiver designed at the Institute of Applied Physics (IAP) at the University of Bern as a replacement of the current MOPI cryogenic receiver. MOPI 5 is a single side band heterodyne receiver designed for the observation of the thermal emission line of ozone at $110.836 \mathrm{GHz}$. It uses state-of-art frond-end components and works at room temperature with a single side band noise temperature of around $550 \mathrm{~K}$. A more thorough description of the MOPI 5 receiver can be found in [18].

\section{A. Spectrometers}

In most radiometers, the radio frequency (RF) signal is downconverted and processed before being analyzed by a spectrometer. Within the spectrometer, different techniques exist to extract the spectral information from the RF signal. With the increase of computational capacity in the last two decades, most spectrometers are now using digital signal processing methods: the RF signal is sampled by a fast analog-to-digital converter (ADC) and fed to a field programmable gate array (FPGA) processor that calculates the spectral components of the signal in real-time.

The Acqiris AC240 was the first commercial fast Fourier transform (FFT) spectrometer used for atmospheric remote sensing. It was developed in 2005 in a collaboration between the company Acqiris and Swiss universities [7], [8]. Since then, it has been widely used in middle atmospheric studies, for ozone, water vapor or temperature profiling around the world.

At the time of the AC240 development, the available FPGA resources did not allow to calculate the FFT on the full $1 \mathrm{GHz}$ bandwidth without small truncation errors. It results in small but noticeable artifacts, especially when higher integration times are needed, which is often the case for ozone profiling instruments. For this reason, the AC240 has now been replaced by the Acqiris U5303 A, which features an improved dynamic range, a larger bandwidth and does not suffer from numerical truncation errors anymore [19].

For this study, we used a U5303 A with a customized spectrometer firmware developed in a collaboration between IAP, Acqiris, and other Swiss universities (simply U5303 from now on). It processes a $1.6 \mathrm{GHz}$ bandwidth and includes some advanced features such as I/Q signal processing with amplitude and phase correction as well as cross correlation that we did not use for our measurements. It also uses a polyphase filter bank (PFB) algorithm, which improves the channel response compared to the FFT algorithm without significant loss of sensitivity [16]. Fig. 1 shows the measured channel responses of the AC240 (FFT) and the U5303 (PFB). Compared to the FFT, which follows very closely the expected $|\operatorname{sinc}(x)|^{2}$ behavior, the PFB shows much faster sidelobe roll-off and a much better channel separation.

Together with the two Acqiris spectrometers, we have used the USRP X310 from Ettus Research, which is a software defined radio (SDR) receiver [20]. For this study, it was used with a customized FFT spectrometer firmware, processing a $200 \mathrm{MHz}$ bandwidth around the ozone line frequency. As an option, the USRP bandwidth can be extended by frequency switching to observe a broader spectral region, and therefore to get profiling capabilities at lower altitudes [21]. For instance, this spectrometer is now operated in our two wind monitoring radiometers observing the ozone emission line at $142 \mathrm{GHz}$ [22]. The main characteristics of the spectrometers used in this study are shown 


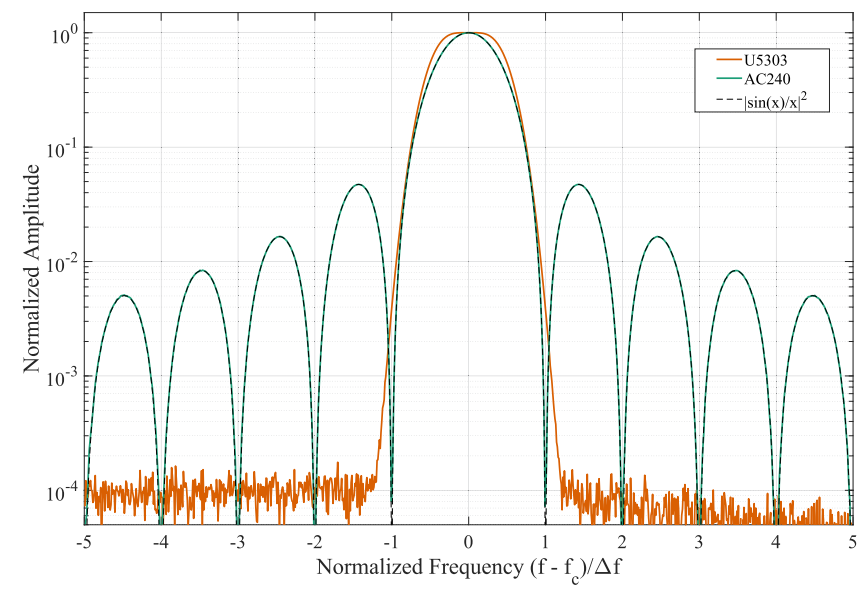

Fig. 1. Measured channel responses of the AC240 and the U5303. The amplitude is normalized to the center channel and the frequency axis is normalized with the channel spacing for both spectrometers. The black dashed line (superimposed to the green line) is the theoretical channel response for an FFT spectrometer with a rectangular window function.

TABLE I

DigitAL SPECTROMETERS USED IN THIS STUDY

\begin{tabular}{|l|c|c|c|c|}
\hline Model & Bandwidth & ADC & $\boldsymbol{\Delta} \mathbf{f}$ & Type \\
\hline AC240 Acqiris & $1000 \mathrm{MHz}$ & 8 bits & $61.04 \mathrm{kHz}$ & FFT \\
\hline U5303 Acqiris & $1600 \mathrm{MHz}$ & $12 \mathrm{bits}$ & $97.66 \mathrm{kHz}$ & PFB \\
\hline USRP Ettus & $200 \mathrm{MHz}$ & 14 bits & $12.21 \mathrm{kHz}$ & FFT \\
\hline
\end{tabular}

in Table I. A more detailed comparison of the spectrometers and how they were connected to the front-end can be found in [16].

\section{MEASUREMENT CAMPAigN}

Between January and June 2019, we operated a test instrument based on the MOPI 5 receiver (denoted simply MOPI 5 from now on) on the roof of one of the buildings of the University of Bern $\left(47^{\circ} \mathrm{N}\right)$ in Switzerland [18]. As back-ends, the AC240, U5303 and USRP were connected in parallel to the instrument and provided simultaneous observations of the atmospheric ozone emission line at $110.836 \mathrm{GHz}$ at an elevation angle of $40^{\circ}$. The radiometer was calibrated by switching continuously between the atmospheric signal, an ambient temperature calibration target and a liquid nitrogen (LN2) cold target. If we neglect the time needed for technical maintenance, MOPI 5 provided more than $350 \mathrm{~h}$ of parallel observations with the three spectrometers. Despite the laborious manual refilling of the liquid nitrogen target, the observations covered a broad range of atmospheric conditions distributed mostly over four months (January to April). An example of the time series recorded during the month of February is shown in Fig. 2.

\section{A. Calibration and Integration}

To investigate the bias between the calibrated spectra of the AC240 and the two other back-ends previously described in [16], we have devised new harmonized calibration and integration routines for the three spectrometers. They use a hot-cold calibration scheme and compute 10 min averaged brightness temperature spectra for each spectrometer. Based on a set of
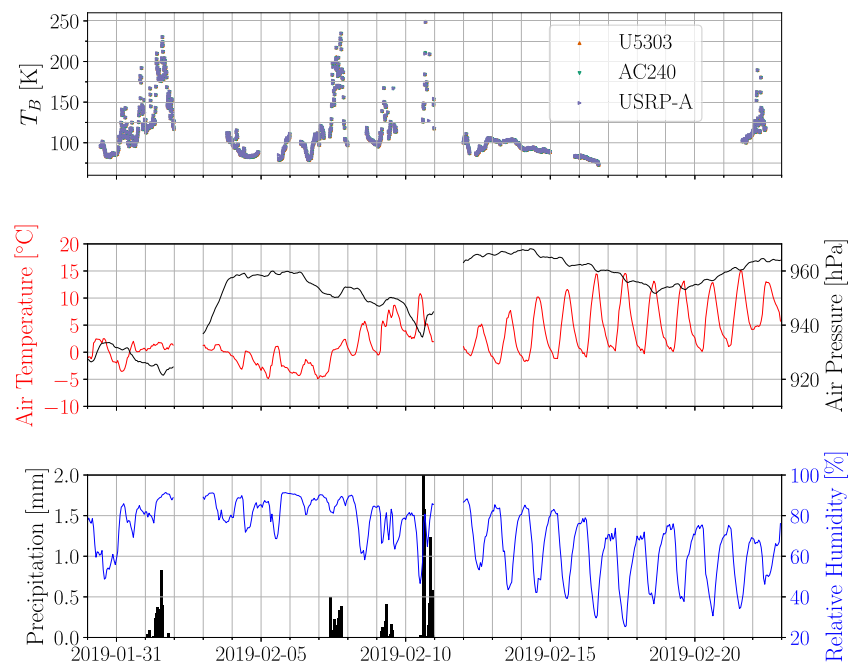

Fig. 2. Time series with $10 \mathrm{~min}$ averaged brightness temperature $\left(T_{B}\right)$ from the 3 spectrometers (top panel), hourly averaged air pressure and temperature (middle panel), and precipitation and relative humidity (bottom panel) recorded in Bern.

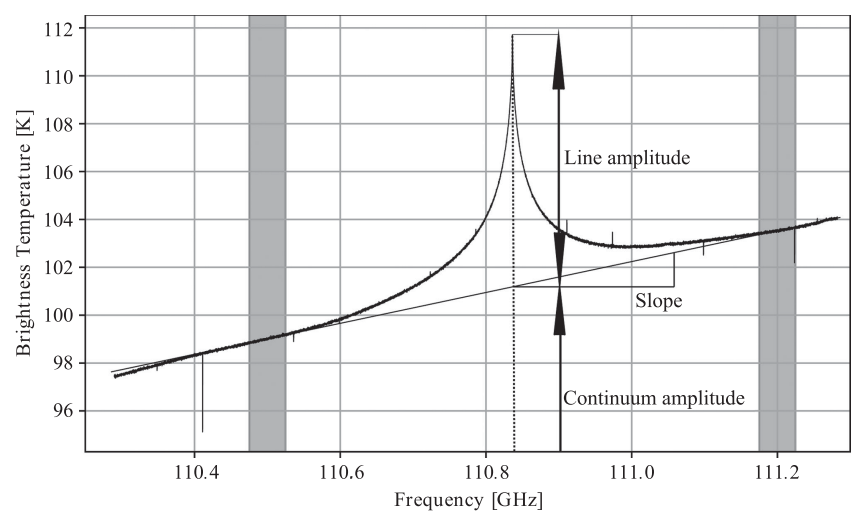

Fig. 3. Sketch of the parameters used for the bias description. The shaded area show the frequency bands used to fit the slope of the spectra. The continuum amplitude $\left(T_{B, c}\right)$ is the brightness temperature at which the fitted slope crosses the line center frequency. The line amplitude $\left(T_{B, l}\right)$ is the difference between the emission peak and the continuum amplitude.

additional parameters (noise temperature spectrum, number of spectra for each target, etc.), this new routine identifies the periods where technical problems occurred on the instrument and flags the corresponding calibrated spectra. It enables one to sort out the spectra of dubious quality before integration.

To improve our understanding of the bias, we decided to investigate the relationship between the bias and the atmospheric opacity. At microwave frequencies increases in tropospheric water vapor are the main cause of increasing opacity, and hence increasing continuum brightness temperature and decreasing stratospheric ozone line strength. In the case of the MOPI 5 observations at $110.836 \mathrm{GHz}$, the oxygen emission band at $118 \mathrm{GHz}$ additionally induces a characteristic slope in the calibrated spectrum, with a brightness temperature increasing towards higher frequencies (see Fig. 3). Therefore, it is possible to compute the tropospheric opacity observed during each calibration cycle from its mean spectral brightness temperature $T_{B, \text { mean }}$ using the method described in [23]. It enables sorting of 

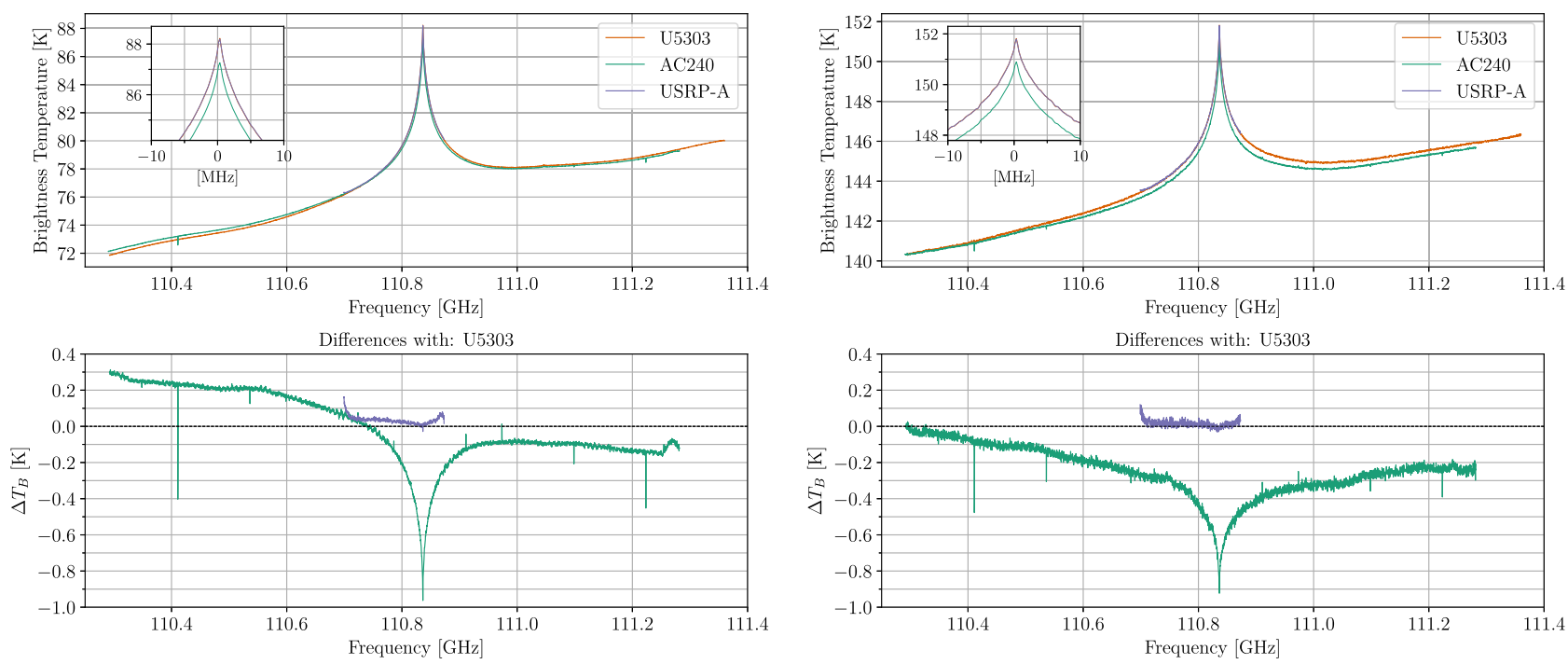

(a)

(b)

Fig. 4. Comparison of the integrated spectra recorded from the three spectrometers in February 2019 for two brightness temperature bins, corresponding to two different atmospheric opacities. The details of the calibration and integration processes are described in the text. (a) Low atmospheric opacity: $T_{B, m e a n}<80 \mathrm{~K}$. (b) High atmospheric opacity: $140 \mathrm{~K}<T_{B, \text { mean }}<150 \mathrm{~K}$.

the calibrated spectra based on their tropospheric opacity before integration. Note that in order to get meaningful results, integrating the same calibration cycles from the three spectrometers is required.

Taking the U5303 as the reference, we computed the mean calibrated brightness temperature from the central channels for each spectrum and collated the calibrated spectra into different brightness temperature $\left(T_{B}\right)$ bins before integration. For the February time period, it resulted in 15 different $T_{B}$ bins, with $T_{B \text {,mean }}$ ranging from 75 to $210 \mathrm{~K}$. Note that we used the same integration periods for the three spectrometers but that the integration time for each $T_{B}$ bin is different as the number of calibrated spectra belonging to each bin is different.

\section{B. Bias Description and Correction}

Fig. 4 shows a comparison of the integrated spectra from the three spectrometers for two different $T_{B}$ bins (out of 15) recorded during the month of February. On the February time period, the integrated spectra from the USRP and the U5303 agree very well, with an averaged brightness temperature difference $\left|\Delta T_{B}\right|<20 \mathrm{mK}$ at the frequency line center $( \pm 25 \mathrm{MHz})$ for all bins. On the contrary, the AC240 shows a systematic negative bias at the line center $\left(\left|\Delta T_{B}\right|>0.4 \mathrm{~K}\right.$ at all bins) and a different spectral slope, which confirms the bias previously observed on the daily spectra by [16]. In addition, the negative bias at the line center and the slope difference on the AC240 are more pronounced at lower tropospheric opacity [see Fig. 4(a)] compared to the higher tropospheric opacity case [see Fig. 4(b)]. The differences shown in Fig. 4 underline the significance of the bias seen on the AC240 but they do not enable identification of its potential sources.

In order to get a better description of the bias observed on the AC240, we computed the three parameters sketched in
Fig. 3, i.e., line amplitude, slope, and continuum amplitude for each integrated spectrum. To study the relationship between the bias and the brightness temperature, we computed these parameters on hourly integrated spectra from the two broadband spectrometers. Compared to the spectra based on $T_{B}$ bins, it allows us to get more data points and a constant integration time $(1 \mathrm{~h})$.

Fig. 5 shows the differences between the parameters evaluated for the U5303 and the AC240 hourly integrated spectra between January and April 2019. It confirms a certain consistency of the bias on the MOPI 5 time series and illustrates its dependency to the brightness temperature. Both the line amplitude and the slope biases follow a close to linear trend with the brightness temperature, which is in accordance with the first observations made from Fig. 4.

Whereas the line amplitude and slope biases have a similar shape, this is not the case for the continuum bias shown in Fig. 5(c). It suggests that the full bias might be a combination of multiple error sources.

Most radiometers assume a linear amplitude transfer characteristic between the calibration loads and the sky observation. It is usually a good approximation but never exactly true and any departure from linearity would result in a bias on the calibrated spectra. From now on, we will refer to this bias simply as "nonlinearities" to distinguish it from the other error sources. As a first order approximation, this nonlinearity is often taken into account by adding a quadratic term on the linear transfer characteristic. It results in a negligible bias when the observed spectrum matches the temperature of one of the calibration loads and in a maximum bias in between. In general, the calibration bias can be derived from a set of brightness measurements at different scene temperatures (as done in [24], [25]). In our case, we can make use of our parallel spectrometers and use the sky measurements directly. As both the U5303 and the USRP 


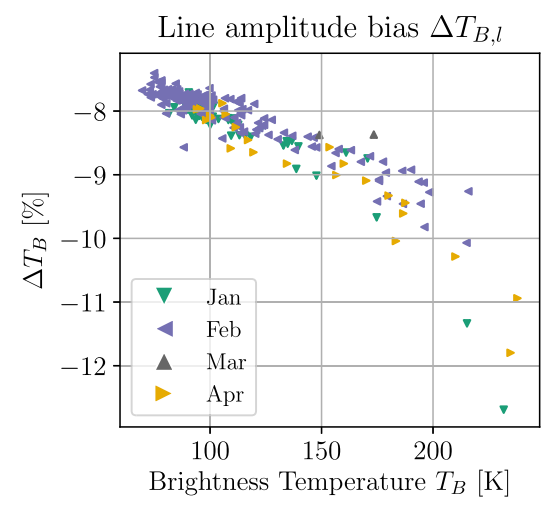

(a)

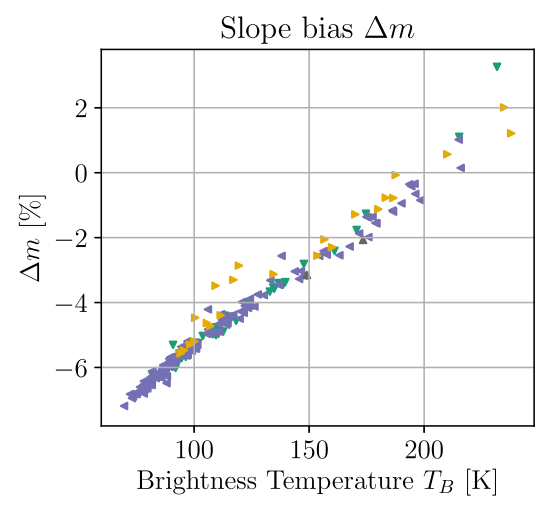

(b)

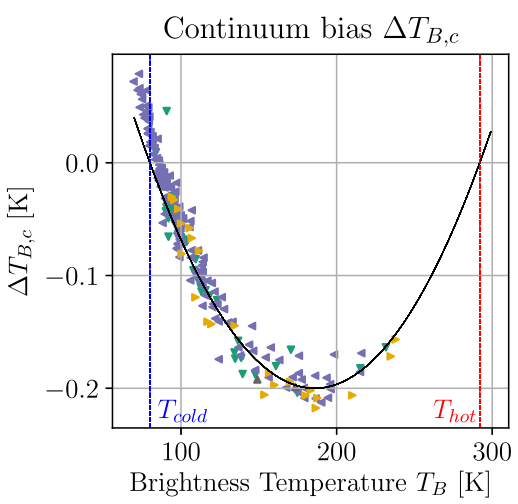

(c)

Fig. 5. Parameters from Fig. 3 computed on hourly integrated spectra between January and April 2019. All three panels show the difference between the parameters computed on the AC240 and the U5303 as a function of the mean brightness temperature of the integrated spectra. (a) Relative differences of the line amplitude bias. It is computed as a fraction of the line amplitude as defined in Fig. 3. For instance for $T_{B}=100 \mathrm{~K}$, the line amplitude is $\approx 10 \mathrm{~K}$ so that the line amplitude bias is $\approx 0.8 \mathrm{~K}$ (or $\Delta T_{B, l}=8 \%$.) (b) Relative differences of the slope bias computed as a fraction of the fitted slope on the U5303. (c) Absolute differences in the continuum bias. The black line shows the modeled quadratic calibration bias that accounts for the nonlinearities.

correspond on the whole range of brightness temperature, we can consider their measurements as the true scene temperature and assume that the continuum bias seen in Fig. 5(c) arises from nonlinearities in the AC240. We can then apply a quadratic correction term to account for its nonlinear amplitude transfer characteristic.

The black line in Fig. 5(c) shows modeled nonlinearities resulting from such a quadratic correction term. It has been derived from the continuum difference between the AC240 and the U5303 $\left(\Delta T_{B, c}\right)$, setting $\Delta T_{B, c}=0$ at $T_{\text {cold }}$ and $T_{h o t}$ and approximating a maximal continuum bias $\Delta T_{B, c}=-0.2 \mathrm{~K}$ in between both calibration load temperatures at

$$
T_{B}=\frac{T_{h o t}+T_{\text {cold }}}{2} \approx 185 \mathrm{~K}
$$

With this simple assumption we are able to reproduce the continuum bias quite well, although our observations do not match the black line in Fig. 5(c) exactly. This might be explained by the fact that $T_{h o t}$ is assumed constant for the whole time series and maybe more importantly, because the mean brightness temperature (abscissa of Fig. 5) is actually computed around the line center, which means that it is a slight overestimation of the full spectrum averaged brightness temperature. Also, we would need more observations at higher brightness temperatures to accurately fit the maximal bias value but as a first estimation, this simple correction is able to correct quite accurately for the nonlinearities on the MOPI 5 measurements.

Although the nonlinearities can account for the continuum bias, they do not explain the line amplitude and slope biases. Looking at Fig. 6(a), we see that even though $T_{B}$ is close to the cold load temperature $\left(T_{B} \approx 80 \mathrm{~K}\right)$, the slope and line amplitude biases are significant, when they should be close to zero if they were only due to nonlinearities. Both seem to follow a close linear relationship with $T_{B}$ with larger uncertainties at higher $T_{B}$. The growing spread of the data points with $T_{B}$ is explained from the growing continuum emission (which is absorbing most of the ozone line features at higher $T_{B}$ ) combined to a lower number of observations at higher $T_{B}$.
The line amplitude and slope biases seem to be the results of a significant "spectral leakage" that would impact the AC240. We still do not understand what exactly could cause such a leakage (see section IV), but it resembles the result of a constant brightness temperature scaling on the AC240. Using a constant scaling factor and incorporating our modeled nonlinearities, we are able to provide a first order correction for the integrated spectra of the AC240

$$
T_{B, \text { corr }}=\frac{1}{(1-\alpha)}\left(T_{B}-\alpha \overline{T_{B}}-\Delta T_{B, c}\right)
$$

where $T_{B, \text { corr }}$ is the corrected brightness temperature spectrum, $\alpha$ is a scaling factor, $\overline{T_{B}}$ is the mean value of the measured spectrum calculated over the entire bandwidth of the AC240, and $\Delta T_{B, c}$ is the nonlinearity correction described above.

Fig. 6 shows the comparisons between the integrated spectra from the U5303 and the AC240 for two atmospheric opacities and three different cases: the original bias (green lines) and the corrected spectra using a constant scaling factor with (purple) and without (grey: $\Delta T_{B, c}=0$ ) the nonlinearity correction. We see that (2) is able to reduce the bias between the AC240 and the U5303. The correction for nonlinearities limits the broadband bias observed when the brightness temperature moves away from the calibration load temperatures, whereas the scaling factor is effective at reducing the slope and the line center biases. We find that a constant scaling factor $(\alpha=8 \%)$ works well for the range of brightness temperatures observed, not only for the month of February but also for the rest of the MOPI 5 observations.

To summarize, it seems that the full bias is a combination of nonlinearities and of a spectral leakage on the AC240. The nonlinearities can be modeled, corrected and it is essentially a broadband contribution to the spectrum which depends on the atmospheric conditions (or on $T_{B, \text { mean }}$ ). On the contrary, the fractional spectral bias seems to remain more or less independent of the atmospheric conditions because a constant scaling parameter is able to correct for the whole range of 

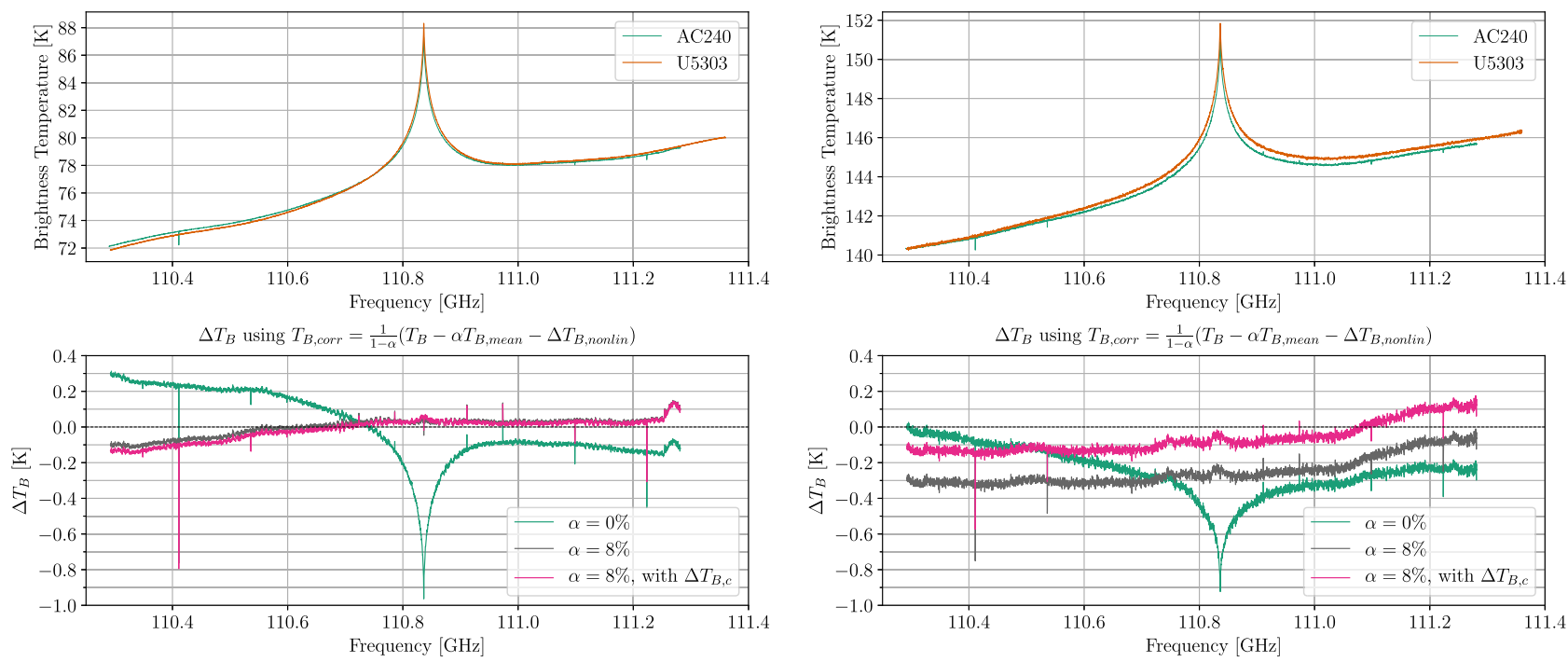

(a)

(b)

Fig. 6. Comparison of the AC240 and the U5303 integrated spectra recorded for two brightness temperature bins, corresponding to two different atmospheric opacities. The bottom panels show the absolute brightness temperature differences between the AC240 spectra before (green line as in Fig. 4) and after it is corrected according to (2). The grey curves do not account for nonlinearities $\left(\Delta T_{B, c}=0\right)$ whereas the purple ones show the full correction with modeled nonlinearities. (a) Low atmospheric opacity: $T_{B, \text { mean }}<80 \mathrm{~K}$. (b) High atmospheric opacity: $140 \mathrm{~K}<T_{B \text {, mean }}<150 \mathrm{~K}$.

atmospheric conditions. The fact that the line amplitude and slope biases evolve with $T_{B}$ (see Fig. 5) might be related to the way these parameters are computed and it will need further studies to validate the dependency of the spectral bias on the atmospheric conditions. Note that the spectral leakage is much more significant for ozone retrievals than the nonlinearities: the former impacts the line amplitude and the slope of the spectrum directly while the latter is essentially a broadband contribution. The line amplitude bias is of particular concern because of its amplitude ( $\approx 8$ to $9 \%$ at the line center) and its immediate impact on the retrieved ozone profile whereas the continuum and slope biases are usually fitted before or during the retrieval process.

\section{Ozone Retrievals}

To study the effect of the observed bias and its approximate correction on the atmospheric profiles, we performed ozone retrievals from the MOPI 5 integrated spectra. At microwave frequencies, the pressure broadening of thermal emission lines enables us to retrieve vertical profiles of atmospheric gases from passive radiometric observations at selected frequencies (e.g., $110.836 \mathrm{GHz}$ for ozone). A retrieval consists at finding the best estimate of the real profile from the shape of the integrated spectra, the measurement errors, a set of a priori information, and a so-called forward model [5]. In microwave radiometry, the forward model is essentially a radiative transfer model that describes the physical relationship between the ozone profiles and the radiometric observations.

As for the calibration routine, we have devised a new routine to retrieve atmospheric ozone profiles from the MOPI 5 measurements at $110.836 \mathrm{GHz}$. As a forward model, we have used the latest version of the atmospheric radiative transfer simulator 2.4
(ARTS) [26]. It is a radiative transfer simulation software with a focus on the microwave region and it also includes some instrumental effects (e.g., channel response) on the simulated spectra. It simulates the atmospheric emission spectra at the radiometer location according to given atmospheric conditions (pressure, temperature, and atmospheric constituents). ARTS provides a large choice of predefined atmospheric scenarios that can be used as inputs for the forward model.

In this study, we used the Fascod climatology, more specifically its mid-latitude winter atmospheric scenario [27]. It is included in the ARTS package and provides all relevant atmospheric variables for the forward model. As a priori ozone profiles, we used a monthly climatology derived from the European Centre for Medium-Range Weather Forecasts (ECMWF) operational reanalysis extended by an AURA/MLS climatology above around $70 \mathrm{~km}$ (similarly as [14]). The spectroscopic parameters were taken from the high-resolution transmission molecular absorption database (HITRAN) [28]. Following the formalism of [5], the optimal estimation method is now integrated within the ARTS package itself, which enables the inversion of the integrated spectra directly within ARTS. This setup was used successfully in [21] for ozone retrievals at $142.175 \mathrm{GHz}$.

Water vapour is the main contributor to opacity at microwave frequencies and is mainly found in the troposphere. Therefore, its contribution to observation of middle-atmospheric ozone is essentially a broadband absorption of radiation emitted by the ozone molecules and it is often removed by a so-called tropospheric correction [23]. In our case, the water vapour continuum absorption was retrieved together with ozone directly from the integrated spectra. Also, due to the lack of standing wave attenuation techniques during the MOPI 5 campaign, we had to include some baseline features to the retrievals (polynomial and sinusoidal) to obtain converging results. As the 

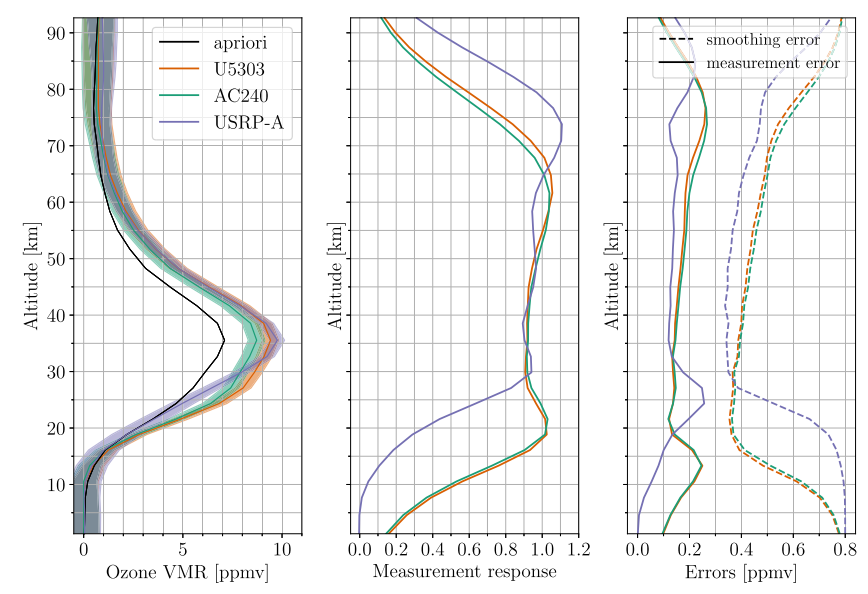

Fig. 7. Example of ozone retrievals from the three spectrometers for the integrated spectra shown in Fig. 4(a). The left panel shows the ozone volume mixing ratio (VMR) with its associated total error, the middle panel shows the measurement response (MR), and the right panel shows the smoothing and measurement errors from the retrievals (see text for details).

integration time was different for each integrated spectrum, it resulted in very different noise levels that had to be taken into account in each retrieval and therefore, the noise covariance matrix was adapted for each brightness temperature bin to get the best possible retrieval. Note that the rest of the retrieval setup (forward model, a priori, spectroscopy, etc.,) was identical for the three spectrometers.

Besides the ozone volume mixing ratio (VMR) profiles, the optimal estimation method provides diagnostic quantities that can be used to derive the averaging kernels (AVKs) or the uncertainty budget of the retrievals (see [5] for more details). The AVKs describe the sensitivity of the retrieval to changes in the true ozone profile and is used to compute the measurement response (MR). The MR is the sum of the AVKs for each altitude and quantifies the amount of information contained in the retrievals that is coming from the actual measurements at a given altitude (the remaining part is coming from the a priori profile). For instance, a MR over 0.8 indicates that the contribution of the radiometric measurements to the "optimal estimated" profile is higher than $80 \%$, i.e., that the a priori contribution is less than $20 \%$. In the case of ozone retrievals, the errors can be separated into three main components: the errors related to the forward model (neglected in the following because they are the same for the three spectrometers), the so-called smoothing error and the measurement error (or retrieval noise). The smoothing error arises from the limited spatial resolution of the microwave radiometer, which prevents resolving fine vertical structure in the profile. The measurement error is linked with uncertainties in the radiometric measurement (e.g., thermal noise). The sum of the smoothing and the measurement error is usually called the total error. A representative example of ozone VMR profiles retrieved from the different spectrometers is shown in Fig. 7 together with their respective measurement responses and retrieval errors characterization. Fig. 8 shows the corresponding AVKs for these specific retrievals, which correspond to the integrated spectra from the first $T_{B}$ bin shown in Fig. 4(a).
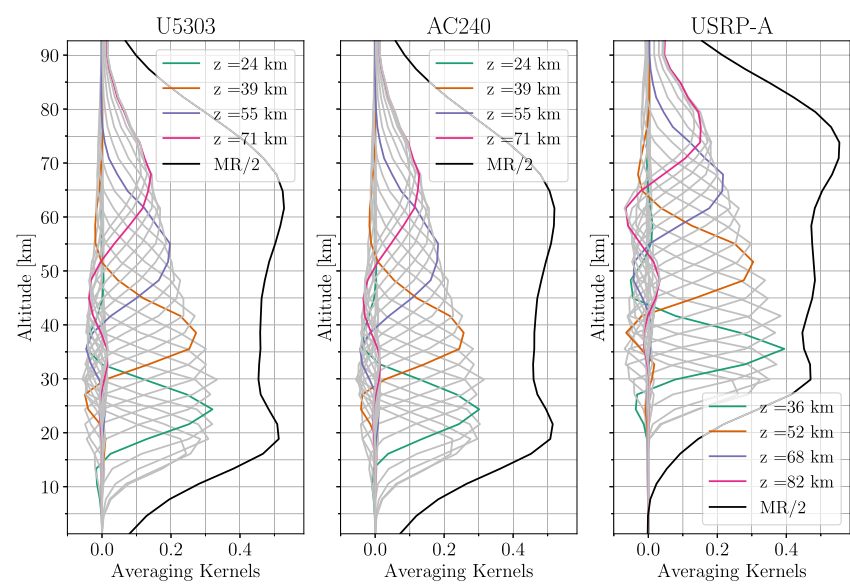

Fig. 8. Averaging kernels (AVKs, grey lines) and measurement responses (MR) corresponding to the ozone retrievals shown in Fig. 7 for the three spectrometers. For clarity, the MR has been divided by 2 (black lines) to be plotted together with the AVKs. We have highlighted some AVKs and labelled them with their corresponding altitude.

\section{Bias on the Ozone Profiles}

To compare the ozone profiles retrieved from the three spectrometers, it is important to underline some of the features shown in Figs. 7 and 8. We see on these figures that the MRs from the AC240 and the U5303 are very similar whereas the MR from the USRP is quite different and seems shifted towards higher altitudes. It is explained by its smaller bandwidth and higher spectral resolution compared to the AC240 and the U5303 (see Table I), which enables the USRP to retrieve ozone profile from $\approx 30$ to $80 \mathrm{~km}$ altitude (MR $\geqslant 80 \%$ is often considered as a lower limit for meaningful retrievals, i.e., when the contribution from the a priori to the final profile is lower than $20 \%$ ), whereas the AC240 and the U5303 have retrievals capabilities from $\approx 20$ to $70 \mathrm{~km}$. The declining MR of the USRP at low altitudes means that from $30 \mathrm{~km}$ downward, the retrieved ozone profile from the USRP is increasingly influenced by the a priori profile and so it explains the strong deviation of the USRP ozone profile from the AC240 and the U5303 below $30 \mathrm{~km}$ (see Fig. 7). Between 30 and $60 \mathrm{~km}$, the retrieved profiles from the USRP and the U5303 are in good agreement (e.g., the mean relative difference between the ozone profiles is less than 3\%), despite some remaining oscillations in some of the retrievals as discussed later. The AC240 and U5303 have similar retrieval capabilities (similar AVKs and MRs) so they can be compared directly. Between 20 and $60 \mathrm{~km}$, the AC240 profile in Fig. 7 shows a strong negative ozone bias compared to the U5303, with close to 0.5 ppmv less ozone retrieved from the $\mathrm{AC} 240$ at the ozone peak around $35 \mathrm{~km}$ altitude.

To evaluate the effect of the spectral and nonlinearity corrections (2) on the ozone profiles, we also retrieved the corrected integrated spectra from the AC240 with the same retrieval routine. Focusing on the ozone difference between the two broadband spectrometers, Fig. 9 shows examples of the relative bias between the $\mathrm{AC} 240$ and the U5303 before and after applying the correction on the AC240 integrated spectra. Fig. 10 shows the relative ozone bias (averaged on $10 \mathrm{~km}$ altitude ranges) of 


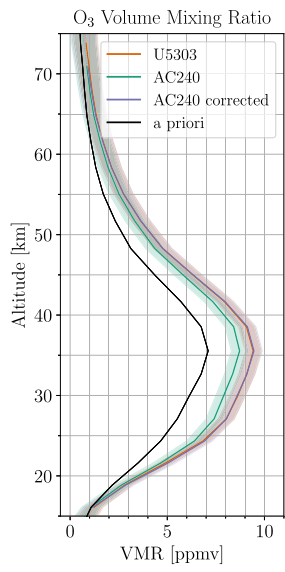

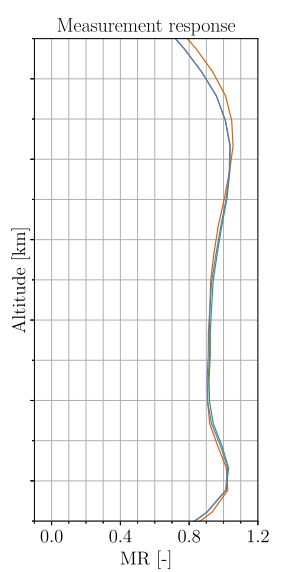

(a)
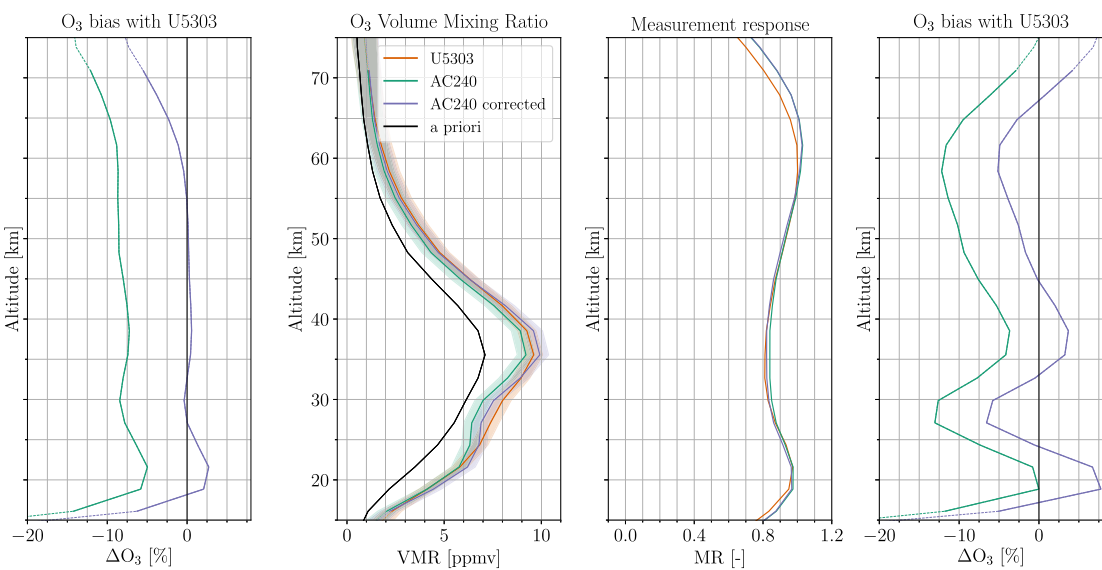

(b)

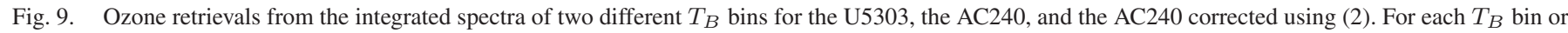
atmospheric opacity case, the left panel shows the retrieved ozone profiles and the middle panel shows the measurement response. The right panel show the relative bias between the ozone profiles retrieved from the AC240 (with and without correction) and the U5303, taken as the reference. (a) Low atmospheric opacity: $T_{B, \text { mean }}<80 \mathrm{~K}$. (b) High atmospheric opacity: $140 \mathrm{~K}<T_{B \text {, mean }}<150 \mathrm{~K}$.

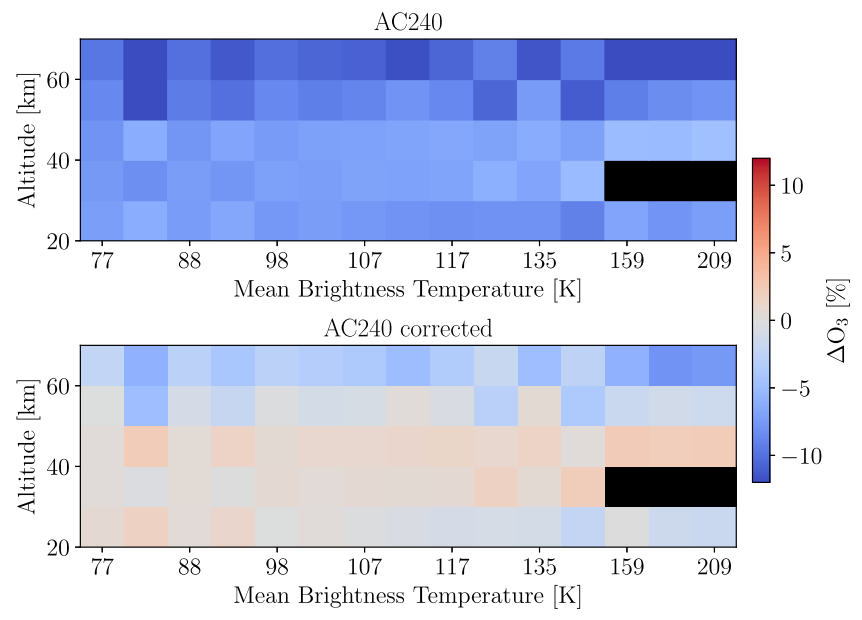

Fig. 10. Mean relative bias in ozone volume mixing ratio compared to the reference spectrometer (U5303) for all altitude ranges and brightness temperature bins (see Section III-A) of the February time period. The bins are labelled using their mean spectral brightness temperature $T_{B, \text { mean }}$. The upper panel shows the original bias of the AC240 spectrometer whereas the lower panel shows the remaining bias after correction with (2) of the AC240 integrated spectra prior to the retrieval. The black squares show the altitude ranges where the measurement responses is constantly lower than $80 \%$.

the AC240 before (upper panel) and after (lower panel) the correction for all $T_{B}$ bins in February. Compared to the U5303, it confirms that the AC240 has a systematic bias of 5\% to $12 \%$ less ozone from 20 to $70 \mathrm{~km}$ altitude. More specifically, the ozone bias is more or less constant between 20 and $50 \mathrm{~km}$ and rises slightly above $50 \mathrm{~km}$. Both Figs. 9 and 10 show that, after correction of the AC240 integrated spectra, the bias between this spectrometer and the U5303 is significantly reduced. It is the case for all $T_{B}$ bins and for all altitude ranges, which is consistent with the systematic negative bias observed on the integrated spectra of the AC240.

As the ozone bias does not strongly depend on the brightness temperature bin (see Fig. 10), Table II presents the mean relative
TABLE II

Mean Relative Bias on the Ozone Profiles With CoRresponding ABSOLUTE VALUE (IN PPMV)

\begin{tabular}{|c|c|c|c|}
\hline Altitude & AC240 - U5303 & AC240 $_{c}-\mathbf{U 5 3 0 3}$ & $\mathbf{A C 2 4 0}^{-} \mathbf{A C 2 4 0}_{c}$ \\
\hline $20-30 \mathrm{~km}$ & $-7.50 \%(-0.52)$ & $-0.21 \%(-0.01)$ & $-7.30 \%(-0.50)$ \\
\hline $30-40 \mathrm{~km}$ & $-6.94 \%(-0.64)$ & $0.70 \%(0.06)$ & $-7.59 \%(-0.71)$ \\
\hline $40-50 \mathrm{~km}$ & $-6.63 \%(-0.41)$ & $1.31 \%(0.08)$ & $-7.79 \%(-0.49)$ \\
\hline $50-60 \mathrm{~km}$ & $-9.23 \%(-0.27)$ & $-1.28 \%(-0.04)$ & $-8.05 \%(-0.23)$ \\
\hline $60-70 \mathrm{~km}$ & $-11.02 \%(-0.17)$ & $-4.11 \%(-0.08)$ & $-7.20 \%(-0.11)$ \\
\hline
\end{tabular}

ozone bias (with corresponding absolute VMR bias [ppmv]) as a function of the altitude range. The effect of the spectral correction is highlighted by showing the ozone profile difference between the AC240 before and after applying the correction $\left(\mathrm{AC} 240-\mathrm{AC} 240_{c}\right)$. As this column does not use the U5303 as the reference, it allows one to see the real impact of (2) on the ozone retrievals. It shows a more or less constant relative bias with altitude and means that the spectral correction has the same effect at all altitudes.

For some retrievals, we noticed oscillations on the resulting VMR profiles (see the right panel of Fig. 9) that we could not remove during the data processing. These oscillations are seen in the data of all three spectrometers and can be explained by a baseline ripple on the integrated spectra, which is mostly caused by standing waves form the LN2 target. In most operational monitoring radiometers this ripple is reduced with an optical path-length modulator, which was not available during this test campaign, resulting in noticeable baselines within the integrated spectra. The spectral noise differences between the spectrometers and the $T_{B}$ bins also made the removal of these oscillations difficult, especially at higher tropospheric opacity, where the ozone line is increasingly absorbed by the water vapor continuum. In our opinion though, these oscillations are artificial and do not call the validity of the bias results into question. However, they may explain why we do not observe a trend in the ozone bias with the $T_{B}$ bins and therefore, further investigation 
is needed to provide any conclusions regarding the evolution of the ozone bias with brightness temperature.

To conclude, despite some technical difficulties during the retrieval processing, the second column of Table II provides a first quantification of the ozone profile bias originating from the AC240 while the fourth column quantifies the effect of the spectral and nonlinearity corrections (2) applied to the integrated spectra. The observed ozone bias is consistent with the spectral bias observed on the integrated spectra of the AC240 and its amplitude is quite large and might explain some of the biases seen in some ozone or water vapour time series derived from the AC240.

\section{DISCUSSION}

The MOPI 5 measurement campaign was quite limited in time and was primarily designed as a test setup for the comparison of digital back-ends. Whereas it provided a unique dataset for the identification of a spectral bias on the AC240, it sets some limitations on the retrieval capabilities and the amount of data collected during this measurement campaign. As an example, the need to manually refill the liquid nitrogen target during the campaign or the large spectral baselines did limit the amount of decent measurements quite significantly and explains why we mostly focused on the month of February in our study. Also, it did not enable us to understand the bias sources, nor to properly validate its successful correction. It results in a limited ability to extrapolate the MOPI 5 results to other instruments, atmospheric lines or different signal-to-noise ratios without further validation. Hereafter, we discuss our hypotheses and attempts to explain and reproduce the spectral bias on the integrated spectrum of the AC240.

\section{A. Origins of the Bias}

The systematic bias seen on the integrated spectra from the AC240 compared to the U5303 seems to have multiple sources. Whereas nonlinearities explains well the continuum difference, it fails to explain the spectral leakage leading to the line amplitude and slope biases. The line amplitude is especially problematic in the case of ozone monitoring because both the slope and continuum biases can be mostly accounted for in the retrieval by the tropospheric correction [23]. In case of nonlinearities, a simple tropospheric correction would still lead to a small error on the frequency line center but this effect should remain small compared to the effect of the spectral leakage.

To this day, we have investigated multiple possible explanations for the observed bias of the line amplitude. Laboratory measurements of the channel response with a swept continuous wave $(\mathrm{CW})$ signal generator in Fig. 1 show that the AC240 follows closely the expected $|\operatorname{sinc}(x)|^{2}$ frequency response of an FFT with a rectangular window function. The convolution of this channel response with simulated ozone emission lines introduces only a negligible bias at the line center and it cannot explain the observed bias of the AC240. This theoretical channel response is also taken into account in the retrieval with ARTS, but its effect on the retrieved ozone profiles is negligible. The channel response measurements were also repeated with a much smaller amplitude of the swept CW signal added to a broadband noise background signal. Also in this case a behavior close to the expected $|\operatorname{sinc}(x)|^{2}$ response was observed, with only a small increase of the side lobe levels for very low signal to background power ratios (SBR).

\section{B. Laboratory Tests}

We attempted to recreate the bias of the AC240 to the U5303, using various power levels and SBRs. A CW signal was coupled with a wideband noise generator (NG) to create an approximation of an atmospheric emission line. The NG output was capped to $800 \mathrm{MHz}$ using a lowpass filter and attenuated until the spectrometer counts were close to the atmospheric measurements. The CW was matched to the peak of a real absorption line and tuned to a frequency located at the center of a channel for both spectrometers simultaneously. In an effort to make the test more representative, the $\mathrm{CW}$ frequency was chosen to be as close to the location of the absorption line in the MOPI 5 measurements as possible. The output was split and read by both spectrometers in parallel. For the test the $\mathrm{CW}$ was cycled ON and OFF every $2 \mathrm{~s}$ and the measurements for each state averaged over the test duration. By computing the relative difference between the $I_{o n}$ (SG ON) and $I_{o f f}$ (SG OFF) spectra for each spectrometer, any power leakage from the SG channel into the other channels should be visible. The relative difference is calculated as follows:

$$
\Delta_{\text {relative }}=\frac{I_{\text {on }}-I_{\text {off }}}{I_{\text {off }}} .
$$

This CW test did not show any spectral leakage from either spectrometer. The same test was repeated at other signal frequencies, power levels, and SBRs with similar results.

It could be still questioned whether these tests with a coherent $\mathrm{CW}$ source are sufficiently representative for the observation of an incoherent atmospheric emission line. For that reason we repeated these measurements with a narrow, band limited noise signal instead of the $\mathrm{CW}$ signal generator. It was generated using a second amplified noise diode, which was followed by a band pass filter with a $3 \mathrm{~dB}$ bandwidth of either 5 or $10 \mathrm{MHz}$. A programmable $70 \mathrm{~dB}$ step attenuator was used to switch between the "ON" and "OFF" cases, which were then added to the broadband noise background. The amplitude of the broadband noise background could be also changed with a second programmable attenuator to mimic the total power calibration of a real radiometer with a hot and a cold calibration load. Fig. 11 shows an example of a U5303 spectra for the background signal with and without the added narrow band noise, as well as with the $6 \mathrm{~dB}$ higher level for the "hot" calibration.

Fig. 12 shows the relative difference between the "ON" by "OFF" states of the band limited noise source calculated with (3). Outside of the narrow band signal the level of the relative difference remains close to zero for the U5303, whereas it increases noticeably for the AC240. This, along with a minor negative offset of the AC240 at the signal peak, is an indication of spectral leakage in the AC240. Given that we did not see this offset during the previous tests with $\mathrm{CW}$ signals, the overall increase in signal power (now spread across a multitude of channels) must be the key difference. We tested this by changing 


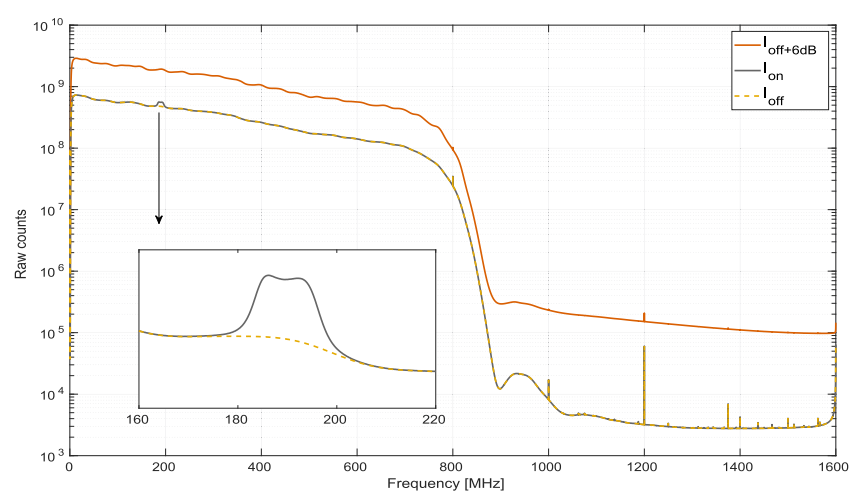

Fig. 11. Example for the three different kinds of spectra recorded during laboratory tests, showing U5303 only.

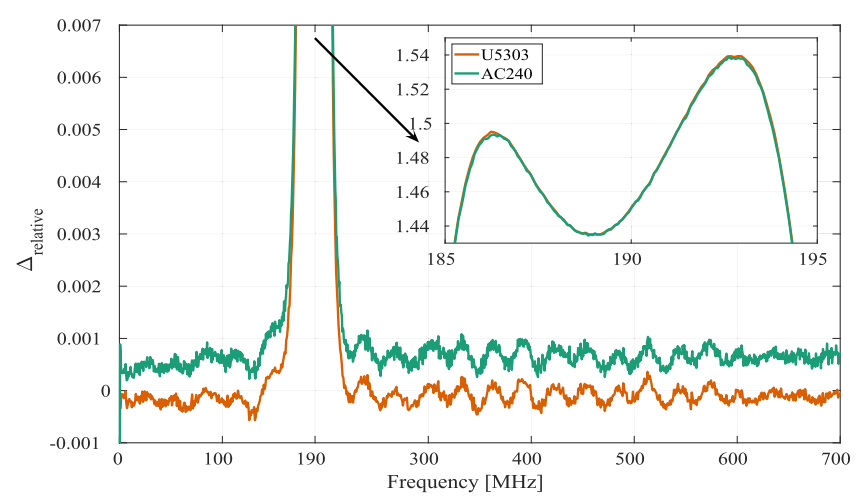

Fig. 12. Relative difference as computed with equation (3) for both U5303 and AC240. Signal frequency: $190 \pm 5 \mathrm{MHz}$.

both the amplitude and width of the signal and could see that the magnitude of the spectral leakage could be reduced by either reducing the width or amplitude of the signal. While this is an indication for the origin of the bias, the magnitude of the leakage is still very small compared to the apparent correction factor for MOPI 5. It should also be considered that the SBR of the test was significantly higher $(\approx 1.5)$ than for the MOPI 5 measurement campaign $(0.05$ or lower).

As previous tests only considered two spectra for comparison, the third test was designed to mimic the calibration process more closely. When considering a "hot" $\left(I_{o f f+6 \mathrm{~dB}}\right)$ spectrum in addition to a "cold" $\left(I_{o f f}\right)$ calibration spectrum and a synthetic atmospheric spectrum $\left(I_{o n}\right)$, a "calibrated" spectrum can be produced by

$$
I_{c a l}=\frac{I_{o n}-I_{o f f}}{I_{o f f+6 d B}-I_{o f f}} .
$$

When comparing the $I_{\text {cal }}$ spectra of the AC240 to the U5303 an offset of roughly $-3 \%$ was visible in the narrow band signal which is consistent with the idea that power is leaking into other channels. Signal amplitude and width (by substituting a smaller bandpass filter) were varied to determine the effect on the offset. Neither change had a noticeable effect on the signal offset in percentage terms. However, the offset between AC240 and U5303 away from the signal changed. A noticeable, but small positive offset of the AC240 from the U5303 was observed in the measurement with the highest SBR $(\approx 0.17)$, but disappeared for all other measurements. Possibly, the reduction in power and width of the signal reduced the magnitude of the spectral leakage to an extent where it was no longer noticeable. When examining the relative difference between only the "hot" and "cold" spectra, using (3) we could see a negative offset across the whole spectrum of similar magnitude as seen in the "calibrated" spectra. Meaning the offset in these tests could be caused by a nonlinearity of the AC240 instead of power leakage, however more tests are required to further investigate this effect and its relation to the spectral bias seen during the MOPI 5 measurement campaign.

\section{CONCLUSION}

The main goal of this contribution was to compare the influence of different digital spectrometers on passive microwave observations of middle-atmospheric ozone. It further investigated a systematic bias previously observed between the calibrated spectra recorded by the widely used AC240 and the more recent U5303 and USRP X310 digital spectrometers. The parallel observations obtained during a measurement campaign confirmed that the AC240 was consistently biased compared to the other two spectrometers. The bias is made up of a lower emission line amplitude and a different spectral slope compared to the U5303 which seems to be the results of a spectral leakage on the AC240. Attempts to reproduce the spectral bias in the laboratory were inconclusive. While we could find some evidence of spectral leakage of the AC240, its observed effect was too small to produce an offset matching the bias observed in the MOPI 5 measurements. Further tests are planned so the root cause of the bias can be determined. In addition, we have identified nonlinearities on this spectrometer, which induce dependency of the bias to the weather conditions (i.e., on the atmospheric opacity). We showed that the observed bias can be reduced significantly with a simple correction consisting of a constant scaling factor and by accounting for the nonlinearities.

We further investigated the effect of the bias on the ozone mixing ratio profiles by performing retrievals with the integrated spectra recorded by the three spectrometers. We observed a negative bias between 6.5 and $11 \%$ on the ozone profiles retrieved from the AC240 compared to the U5303 between 20 and $70 \mathrm{~km}$ altitudes. We also showed that the spectral correction applied on the integrated spectra can significantly reduce the ozone profile bias of the AC240.

The bias observed on the ozone profiles is considerable and might have large impacts on the time series recorded with the AC240. It could help explain the biases already reported with this spectrometer, not only for ozone, but also for water vapour observations. As the origin of the bias is not fully understood yet, further investigation is necessary before implementing the correction for other instruments or atmospheric lines. The AC240 is currently being replaced in several microwave radiometers and we are preparing parallel measurements with AC240/U5303 
on a $142 \mathrm{GHz}$ ozone radiometer and with AC240/USRP on a $22 \mathrm{GHz}$ water vapour radiometers of NDACC. It should provide the required validation to extrapolate our approximate correction to the rest of the NDACC instruments that have used, or are still using, the AC240.

\section{ACKNOWLEDGMENT}

The authors would like to thank J. Hagen for his help to the retrievals as well as N. Jaussi, A. Luder, and T. Plüss for their technical expertise during the measurement campaign and the laboratory work. The authors also acknowledge the numerous developers that contributed to the free and open source tools used for the data analysis and visualization, in particular xarray [29], Matplotlib [30], Typhon, pyretrievals, and the ARTS community [26]. Eric Sauvageat, Klemens Hocke, and Axel Murk would like to acknowledge the support of the Oeschger Centre for Climate Change Research (OCCR).

\section{REFERENCES}

[1] Y. Calisesi, "The stratospheric ozone monitoring radiometer SOMORA: NDSC application document," IAP Res. Rep, vol. 11, pp. 53-54, 2003.

[2] G. E. Nedoluha, R. M. Bevilacqua, R. M. Gomez, D. L. Thacker, W. B. Waltman, and T. A. Pauls, "Ground-based measurements of water vapor in the middle atmosphere," J. Geophysical Res.: Atmos., vol. 100, no. D2, pp. 2927-2939, 1995.

[3] R. M. Gomez, G. E. Nedoluha, H. L. Neal, and I. S. McDermid, "The fourth-generation water vapor millimeter-wave spectrometer," Radio Sci., vol. 47, no. 1, pp. 1-11, 2012.

[4] R. Rüfenacht, N. Kämpfer, and A. Murk, "First middle-atmospheric zonal wind profile measurements with a new ground-based microwave doppler-spectro-radiometer," Atmospheric Meas. Techn., vol. 5, no. 11, pp. 2647-2659, 2012

[5] C. D. Rodgers, "Inverse methods for atmospheric sounding: Theory and practice," World Sci., vol. 2, pp. 65-80, 2000.

[6] I. Petropavlovskikh, S. Godin-Beekmann, D. Hubert, R. Damadeo, B. Hassler, and V. Sofieva, "SPARC/IO3C/GAW report on long-term ozone trends and uncertainties in the stratosphere," Tech. Rep., Feb. 2019, 9th Assessment Report of the SPARC Project, Published by the International Project Office at DLR-IPA. Also: GAW Report No 241; WCRP Report 17/ 2018. [Online]. Available: https://elib.dlr.de/126666/

[7] A. O. Benz et al., "A broadband FFT spectrometer for radio and millimeter astronomy," Astron. Astrophys., vol. 442, no. 2, pp. 767-773, 2005.

[8] S. Müller, A. Murk, C. Monstein, and N. Kämpfer, "Intercomparison of digital fast fourier transform and acoustooptical spectrometers for microwave radiometry of the atmosphere," IEEE Trans. Geosci. Remote Sens., vol. 47, no. 7, pp. 2233-2239, Jul. 2009.

[9] G. E. Nedoluha, R. M. Gomez, B. C. Hicks, J. Helmboldt, R. M. Bevilacqua, and A. Lambert, "Ground-based microwave measurements of water vapor from the midstratosphere to the mesosphere," $J$. Geophysical Res.: Atmos., vol. 116, no. D2, pp. 9-10, 2011.

[10] B. Tschanz, C. Straub, D. Scheiben, K. A. Walker, G. P. Stiller, and N. Kämpfer, "Validation of middle-atmospheric campaign-based water vapour measured by the ground-based microwave radiometer MIAWARA-C," Atmospheric Meas. Techn., vol. 6, no. 7, pp. 1725-1745, 2013.

[Online]. Available: https://amt.copernicus.org/articles/6/1725/2013/

[11] L. Bernet et al., "Ground-based ozone profiles over central Europe: Incorporating anomalous observations into the analysis of stratospheric ozone trend," Atmospheric Chem. Phys., vol. 19, no. 7, pp. 4289-4309, 2019. [Online]. Available: https://acp.copernicus.org/articles/19/4289/2019/

[12] P. F. Orte et al., "Analysis of a southern sub-polar short-term ozone variation event using a millimetre-wave radiometer," Annales Geophysicae, vol. 37, no. 4, pp. 613-629, 2019. [Online]. Available: https://angeo. copernicus.org/articles/37/613/2019/

[13] M. De Mazière et al., "The network for the detection of atmospheric composition change (NDACC): History, status and perspectives," Atmospheric Chem. Phys., vol. 18, no. 7, pp. 4935-4964, 2018. [Online]. Available: https://acp.copernicus.org/articles/18/4935/2018/
[14] S. Studer, K. Hocke, M. Pastel, S. Godin-Beekmann, and N. Kämpfer, "Intercomparison of stratospheric ozone profiles for the assessment of the upgraded GROMOS radiometer at bern," Atmospheric Meas. Techn. Discuss., vol. 6, pp. 6097-6146, 2013.

[15] E. Maillard Barras et al., "Study of the dependence of long-term stratospheric ozone trends on local solar time," Atmospheric Chem. Phys. vol. 20, no. 14, pp. 8453-8471, 2020. [Online]. Available: https://acp. copernicus.org/articles/20/8453/2020/

[16] A. Murk and M. Kotiranta, "Characterization of digital real-time spectrometers for radio astronomy and atmospheric remote sensing," in Proc. Int. Symp. Space THz Technol., Gothenburg, Sweden, vol. 15, 2019.

[17] E. Sauvageat, M. Kotiranta, K. Hocke, R. M. Gomez, G. Nedoluha, and A. Murk, "Comparison of three high resolution real-time spectrometers for microwave ozone profiling instruments," in Proc. 16th Specialist Meeting Microw. Radiometry Remote Sens. Environ., 2020, pp. 1-4.

[18] M. Kotiranta, R. M. Gomez, G. Nedoluha, N. Kämpfer, and A. Murk, "Receiver development for the microwave ozone profiling instrument MOPI 5," in Proc. IEEE Int. Geosci. Remote Sens. Symp., 2019, pp. 8952 8955.

[19] "Enhanced fast Fourier transform application on 12-bit high-speed data acquisition PCIe Card," Accessed: Nov. 5, 2021. [Online]. Available: https: //acqiris.com

[20] "Ettus research, a national instruments brand," Accessed: Nov. 5, 2021 [Online]. Available: https://www.ettus.com/

[21] J. Hagen, A. Luder, A. Murk, and N. Kämpfer, "Frequency-Agile FFT spectrometer for microwave remote sensing applications," Atmosphere, vol. 11, no. 5, pp. 3-6, 2020. [Online]. Available: https://www.mdpi.com/ $2073-4433 / 11 / 5 / 490$

[22] J. Hagen, A. Murk, R. Rüfenacht, S. Khaykin, A. Hauchecorne, and N. Kämpfer, "WIRA-C: A compact 142-GHz-radiometer for continuous middle-atmospheric wind measurements," Atmospheric Meas. Techn., vol. 11, no. 9, pp. 5007-5024, 2018. [Online]. Available: https://amt. copernicus.org/articles/11/5007/2018/

[23] T. Ingold, R. Peter, and N. Kämpfer, "Weighted mean tropospheric temperature and transmittance determination at millimeter-wave frequencies for ground-based applications," Radio Sci., vol. 33, no. 4, pp. 905-918, Jul. 1998.

[24] F. Weng et al., "Calibration of Suomi national polar-orbiting partnership advanced technology microwave sounder," J. Geophysical Res.: Atmos., vol. 118, no. 19, pp. 11-187, 2013.

[25] R. V. Leslie, W. J. Blackwell, A. Cunningham, M. DiLiberto, J. Eshbaugh, and I. A. Osaretin, "Pre-launch calibration of the NASA tropics constellation mission," in Proc. IEEE 16th Specialist Meeting Microw. Radiometry Remote Sens. Environ., 2020, pp. 1-4.

[26] S. A. Buehler, J. Mendrok, P. Eriksson, A. Perrin, R. Larsson, and O. Lemke, "ARTS, the atmospheric radiative transfer simulator - version 2.2, the planetary toolbox edition," Geoscientific Model Develop., vol. 11, no. 4, pp. 1537-1556, 2018. [Online]. Available: https://gmd.copernicus. org/articles/11/1537/2018/

[27] G. P. Anderson, S. A. Clough, F. Kneizys, J. H. Chetwynd, and E. P. Shettle, "AFGL atmospheric constituent profiles $(0.120 \mathrm{~km})$," Air Force Geophysics Lab, Hanscom AFB, MA, USA, Tech. Rep., 1986.

[28] I. E. Gordon et al., "The HITRAN2016 molecular spectroscopic database," J. Quantitative Spectrosc. Radiative Transfer, vol. 203, pp. 3-69, 2017.

[29] S. Hoyer and J. Hamman, "Xarray: ND labeled arrays and datasets in python," J. Open Res. Softw., vol. 5, no. 1, p. 10, 2017.

[30] J. D. Hunter, "Matplotlib: A 2D graphics environment," IEEE Ann. Hist. Comput., vol. 9, no. 3, pp. 90-95, May 2007.

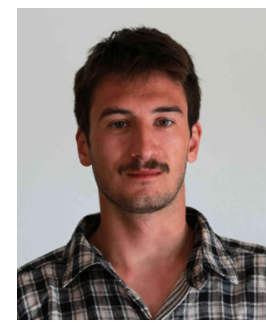

Eric Sauvageat received the M.Sc. (Eng.) degree in energy management and sustainability from Ecole Polytechnique Fédérale de Lausanne, Lausanne, Switzerland, in 2018 and is currently working toward the Ph.D. degree in applied physics with the University of Bern, Bern, Switzerland.

His main research interests include microwave remote sensing and data analysis of atmospheric processes, with a focus on middle-atmospheric ozone. 


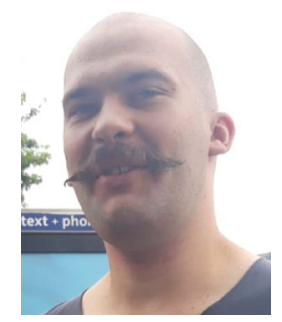

Roland Albers received the B.Eng. degree in aerospace engineering from Brunel University, London, U.K. in 2015, and the M.Sc. degree in astronautics and space engineering from Cranfield University, Milton Keynes, U.K., in 2016. He is currently working toward the Ph.D. degree in applied physics with the University of Bern, Bern, Switzerland.

He worked in the space industry on quasi-optical components. His research interests include spaceborne radiometry, hardware design, and calibration.

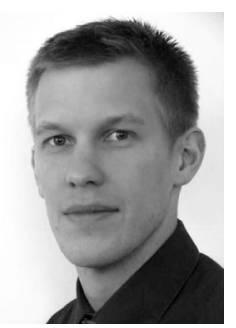

Mikko Kotiranta received the M.Sc. (Tech.) degree (Hons.) in electrical engineering from the Helsinki University of Technology, Espoo, Finland, in 2008 , and the Ph.D. degree in physics from the Goethe University of Frankfurt, Frankfurt am Main, Germany, in 2013.

$\mathrm{He}$ is currently with the Institute of Applied Physics, University of Bern, Bern, Switzerland, where he is engaged in the development of quasioptical components and systems at millimeter and submillimeter wavelengths for atmospheric and as-

trophysical research.

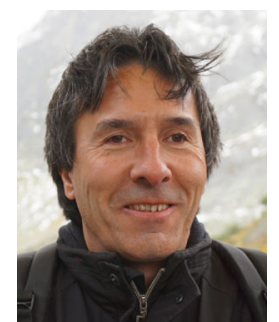

Klemens Hocke received the venia legendi in atmospheric physics from the University of Bern, Bern, Switzerland, in 2011.

$\mathrm{He}$ is the leader of the Atmospheric Processes Group, Institute of Applied Physics, University of Bern. His interests include atmospheric dynamics, remote sensing, and data analysis of atmospheric water and stratospheric ozone.

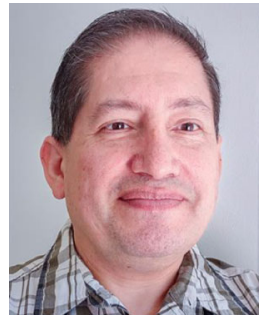

R. Michael Gomez received the B.S. degree in physics from George Mason University, Fairfax, VA, USA, in 1991

$\mathrm{He}$ is the Chief Engineer with the U.S. Naval Research Laboratory's microwave spectroscopy program. For the past 30 years, he has been developing microwave instrumentation for the investigation of atmospheric chemistry and dynamics. His research interests include hardware design, remote sensing applications, and atmospheric processes.

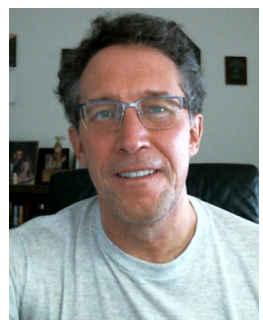

Gerald E. Nedoluha received the B.S. degree in physics from U.C. Berkeley, Berkeley, CA, USA, in 1983, and the Ph.D. degree in physics from the University of Illinois, Champaign, IL, USA, in 1990.

$\mathrm{He}$ is the Head of the Middle Atmospheric Physics Section with the U.S. Naval Research Laboratory, Washington, D.C., USA. He is also the Principle Investigator of a ground-based microwave spectroscopy program to conduct long-term measurements of $\mathrm{H}_{2} \mathrm{O}$, $\mathrm{ClO}$, and $\mathrm{O}_{3}$ in the middle atmosphere.

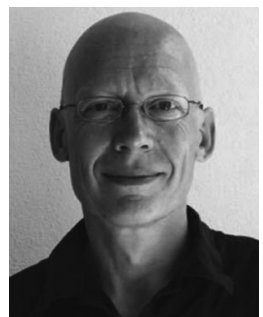

Axel Murk received the M.Sc. degree in physics from the Technical University of Munich, Munich, Germany, in 1995, and the Ph.D. degree in physics from the University of Bern, Bern, Switzerland, in 1999.

Since then, he has been involved in the development and characterization of millimeter- and submillimeter-wave instrumentation for different ground-based and space-borne projects. Since 2018, he is leading the IAP Microwave Physics Division with the University of Bern. His research interests include digital real-time spectrometers and the radiometric calibration of remote sensing instruments. 